\title{
THE RIPPLE EFFECT OF COVID-19 PANDEMIC IN HEALTHCARE
}

\author{
Aaminah Najmus Sahar ${ }^{*}$ 可 (iD) \\ ${ }^{* 1}$ Department of Pharmacy Practice, Deccan School of Pharmacy (DSOP), OU, Hyderabad-500001, \\ Telangana, India
}

DOI: https://doi.org/10.29121/granthaalayah.v8.i12.2020.2518

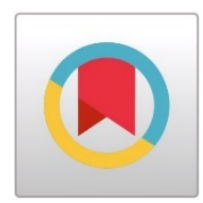

Article Type: Research Article

Article Citation: Aaminah Najmus Sahar. (2020). THE RIPPLE EFFECT OF COVID-19 PANDEMIC IN

HEALTHCARE. International Journal of Research -GRANTHAALAYAH, 8(12), 30-35.

https://doi.org/10.29121/granthaa layah.v8.i12.2020.2518

Received Date: 23 November 2020

Accepted Date: 24 December 2020

Keywords:

Covid-19 Pandemic

Healthcare

Ripple Effect

Mental Health

Antimicrobial Resistance (Amr)

Antimicrobial Stewardship

Programs (Asp)

Addictions, Substance Use Disorders (Sud's)

\section{ABSTRACT}

The COVID-19 Pandemic has disrupted life and changed what we knew as normal, for the past 9 months. Although the first case of COVID-19 was identified in Wuhan, China in mid-December 2019, it was coined as a Pandemic by WHO in March 2020. The COVID-19 Pandemic has impacted each and every one and caused a ripple effect in all areas of life, the healthcare is no exception. With its novel nature, limited information, lack of resources, risky working conditions, and high spikes in cases, it has caused a massive overload on the healthcare systems. It has caused mental health disturbances not just in the frontline workers and COVID-19 survivors but also in the general public owing to uncertainty, isolation, fear, anxiety, and misinformation.

In addition to this, there has also been an increase in the prescription of antibiotics which stems from the inability to examine the patient physically and obtain samples for cultures as consultations are scheduled through telemedicine. Antimicrobial resistance (AMR) is considered to be further potentiated during the Pandemic as Antimicrobial stewardship programs (ASP) have been disrupted and there is an increase in hospital admissions and intubations of patients. Measures like lockdown and travel restrictions placed to curb the spread of the virus have negatively impacted individuals with addictions and substance use disorders (SUD's) due to inability to access de-addiction centers, increased isolation, and inability to obtain toxicants which lead to the use of adulterated substances and in some cases withdrawal symptoms. The lockdown has also caused delays in the treatment of chronic and co-morbid diseases like Diabetes, Cardiovascular diseases, Cancer, Dialysis leading to poor disease management and progression of the disease. It has also impacted regular vaccination schedules and periodic health checkups leading to late diagnosis of diseases.

\section{INTRODUCTION}

COVID-19 was first identified in Wuhan, Hubei Province of China in mid-December 2019. It is a novel virus belonging to the strain of Coronaviruses. Earlier it was called n-COV (novel Coronavirus) disease, later the term COVID-19 (Coronavirus disease-2019) was coined by the WHO. COVID-19 is caused by the SARS-COV-2 virus. The disease spread rapidly from China to the rest of the world and was termed a Pandemic in March 2020.

(C) 2020 The Author(s). This is an open access article distributed under the terms of the Creative Commons Attribution License, which permits unrestricted use, distribution, and reproduction in any medium, provided the original author and source are credited. 
The COVID-19 Pandemic has caused 1.48 million deaths and 63.9 million confirmed cases worldwide as of now, with the cases increasing with each passing day. In addition to the millions of positive cases and deaths worldwide, leading to a massive overload on the hospitals and healthcare, COVID-19 Pandemic has also caused a ripple effect in healthcare.

The Ripple effects of COVID-19 Pandemic in healthcare are enlisted below.

\subsection{IMPACT OF COVID-19 PANDEMIC ON MENTAL HEALTH-}

The COVID-19 Pandemic has caused restlessness and mental health abnormalities all over the earth, from the general population to frontline workers to individuals who themselves/their families have tested positive for COVID19.

Coronavirus Disease (COVID)-19 pandemic is said to be harming the mental health of the general population through a range of suggested mechanisms: fear, uncertainty, and anxiety; social distancing/isolation; loneliness; and economic repercussions.[1] It has especially impacted the mental health of the vulnerable population- children, elderly, poor and marginalized, individuals with pre-existing mental health conditions, individuals with substance use disorders (SUD's). Depression, anxiety, insomnia, fear, PTSD, addiction to the internet and gambling, drug abuse, child abuse, domestic violence, and self-harm are some of the tragic consequences of the COVID-19 Pandemic.

Frontline workers have been experiencing emotional overload due to several reasons, such as shortage of suitable personal protective equipment, reduction in human resources and relentless work shifts, fear of becoming infected and infecting relatives, high mortality rates, grieving the loss of patients and colleagues, separation from families.[1] According to a study conducted in China, among the first-line medical staff of a Tertiary Infectious Disease Hospital for COVID-19, the incidence of anxiety and post-traumatic symptoms in female medical staff was higher than that in male, and in nurses more represented than that in doctors [2]

A study conducted in Italy, found that COVID-19 recovered patients suffered from PTSD (28\%), depression (31\%), anxiety (42\%), insomnia (40\%), 20\% for OC symptoms. Overall, 56\% scored in the pathological range in at least one clinical dimension. [3]

Severe Acute Respiratory Syndrome (SARS) in 2003 had similarly contributed to increased anxiety, mood, and thought disorders, adjustment disorders, and post-traumatic stress disorders (PTSD), resulting, in extreme cases, in suicidal behaviors (e.g., suicidal ideation, suicide attempts, and actual suicide)(1)According to a recent study from the Well Being Trust, the high levels of stress, isolation and unemployment due to the COVID-19 pandemic could cause up to 75,000 "deaths of despair" related to deaths to drug, alcohol, and suicide [4]

There has been a significant impact of the COVID-19 Pandemic on mental health and thus it's important to assess the psychopathology of COVID-19 survivors, frontline workers, and the general public, particularly individuals with pre-existing mental health conditions. It is important to monitor the mental health of the above-mentioned individuals over a period to avoid an epidemic of mental health disorders. To maintain good mental health strategies like stress management and coping by structuring activities and maintaining a routine, linking patients to social and mental health services, and counseling patients to seek professional mental health assistance when needed have been implemented. Since media reports can be emotionally disturbing, contact with pandemic-related news should be monitored and limited. As for health care providers, the health care systems will need to address the stress on individual providers and general operations by monitoring reactions and performance, altering assignments and schedules, modifying expectations, and creating mechanisms to offer psychosocial support as needed. Education and training regarding psychosocial issues should be provided to health system leaders, first responders, and health care professionals. [5]

\subsection{IMPACT OF COVID-19 PANDEMIC ON ANTIMICROBIAL RESISTANCE (AMR) -}

Antimicrobial Resistance (AMR) occurs when bacteria, viruses, fungi, and parasites change over time and no longer respond to medicines. It results in infections harder to treat and increasing the risk of disease spread, severe illness, and death. Such modified micro-organisms that are resistant to treatment are known as superbugs.

According to WHO, Antimicrobial resistance is considered an impending pandemic and the biggest threat to global health. It is predicted that AMR will result in the death of 10 million people each year by 2050 . In common bacterial infections, including urinary tract infections, sepsis, sexually transmitted infections, and some forms of 
diarrhea, high rates of resistance against antibiotics frequently used to treat these infections have been observed worldwide, indicating that we are running out of effective antibiotics. Similarly, antibiotic-resistant Mycobacterium tuberculosis strains are threatening progress in containing the global tuberculosis epidemic. Antiviral drug resistance is an increasing concern in immunocompromised patient populations, where ongoing viral replication and prolonged drug exposure lead to the selection of resistant strains. Resistance has developed to most antivirals including antiretroviral (ARV) drugs. Increased resistance in malarial parasites and fungi have also been noted.[6]

The COVID-19 Pandemic has further potentiated the threat of AMR, as many patients admitted to the hospital with COVID-19 receive antibiotics to keep secondary bacterial infections in check.[7] A review of studies published on hospitalized COVID-19 patients identified that while $72 \%(1450 / 2010)$ of patients received antibiotics, only $8 \%$ $(62 / 806)$ demonstrated superimposed bacterial or fungal co-infections. [8] WHO also reports that azithromycin is being widely used with hydroxychloroquine although it is not yet recommended outside of COVID-19 clinical trials. [9] In addition to this, the lockdown and use of telemedicine increased antibiotic use. Increased hospital admissions also increased the risk of transmission of multi-drug resistant bacteria. Disruptions to health services during the pandemic are causing interruptions to treatments, such as for tuberculosis and human immunodeficiency virus, which can also lead to selection for drug resistance. Similarly, disruption to vaccination services can increase the risk of infection, potentially leading to an overuse of antimicrobials[10] Another potential threat to AMR is the wide use of biocidal agents for environmental and personal disinfection, including in non-health-care settings. Low-level exposure to biocidal agents can select for drug-resistant strains and enhance the risk of cross-resistance to antibiotics,[11] particularly those that treat Gram-negative bacteria.[12] Higher concentrations of these biocides are likely to be detected in wastewater treatment plants and receiving waters. This may increase levels of AMR in the environment, posing a human health risk for individuals exposed to these environments. [13]

To fight AMR in the COVID-19 Pandemic the latest update of the interim guidelines for clinical management of COVID-19 Pandemic incorporates antimicrobial stewardship activities. The guidelines do not recommend antibiotic therapy or prophylaxis for patients with mild or moderate COVID-19 unless signs and symptoms of a bacterial infection exist. The guidelines further state that empiric antibiotic bacterial pneumonia treatment can be considered in older people residing in long-term care facilities and children younger than five years with moderate COVID-19. As these are non-hospitalized patients, antibiotics within WHO's AWaRe (access, watch, reserve) classification of antibiotics categorized as access, such as co-amoxicillin, should preferably be administered.[10]

To incorporate Antimicrobial stewardship into the Pandemic response it is necessary to -

- Increase clinical competence (ability to identify signs and symptoms of severe COVID-19 and that of a superimposed bacterial or fungal disease; eliminate unnecessary antibiotic use including daily deescalation; evaluate the need for medical devices and others that increase the chances of health-careassociated infections and antibiotic use, and implement strict infection prevention and control measures) among health workers treating COVID-19 patients.

- Ensure the continuity of essential health services and regular supply of quality-assured and affordable antimicrobials including antiretroviral and tuberculosis drugs, and vaccines.

- Reduce the turnaround time of COVID-19 testing by improving testing methods and expanding testing facilities, especially for presumed patients, to reduce the urge to initiate antibiotics.

- Exercise maximum caution in the use of biocides for environmental and personal disinfection and prioritize biocidal agents without or with a low selection pressure for antibiotic resistance.

- Address gaps in research (rapid and affordable diagnostic tests that differentiate between bacterial and viral respiratory tract infections; the short- and long-term impact of the wide use of biocides for environmental and personal disinfection including cross-resistance to antimicrobials; and potential alternatives for sustainable environmental and personal disinfection) to ensure that antimicrobial stewardship activities become an integral part of the pandemic response and beyond. [10]

In addition to this, the following Antimicrobial stewardship activities should also be incorporated-Auditing the treatment plan with intervention and feedback, formulary restriction and pre-authorization, educating health care providers about antimicrobial stewardship, switching from IV to PO therapy when possible as this could shorten the hospital stay and reduce risk of transmission of bacteria, engagement with microbiology laboratories and infection prevention programs. [14] 
Aaminah Najmus Sahar

Thus, by taking adequate precautions the global threat of AMR could be prevented from further escalation during the COVID-19 Pandemic.

\subsection{IMPACT OF COVID-19 PANDEMIC ON INDIVIDUALS WITH ADDICTIONS, SUBSTANCE USE DISORDERS(SUD's)-}

The COVID-19 Pandemic has impacted the entire society but those who are significantly impacted yet have been neglected are individuals with addictions and SUD. With the lockdown and isolation, they may experience-(a) changes in levels of drug use-an increase is often seen as a reactive behavior to the negative impact of disasters;

(b) a shift to other substances if access to those previously used become limited due to lockdown;

(c) a relapse, if they had already recovered from alcohol/drug addiction due to the inability to access the deaddiction centers in lockdown.[1]

Several individuals have experienced withdrawal symptoms (which was fatal in some cases) due to the sudden unavailability of alcohol and other toxicants. In addition to this, there is an increased risk of overdose as well for addicted people in home isolation, as there is no one present to inject them with Naloxone. Another risk is the use of adulterated and contaminated drugs and needles by individuals with SUD, as the drug supply has been significantly impacted by lockdown and travel restrictions.[15] Individuals with addictions and SUD's are also at a greater risk of severe COVID-19 due to underlying physical comorbidities (lung diseases, cardiovascular diseases, HIV, viral infections, immunosuppression, etc.) and social factors (homelessness, uninsured, economic difficulties)[16]

To manage the addiction epidemic during the COVID-19 Pandemic measures like access to de-addiction centers, telemedicine, social support, and virtual peer groups are important.

\subsection{IMPACT OF COVID-19 PANDEMIC ON CHRONIC AND CO MORBID DISEASES-}

The COVID-19 Pandemic has negatively impacted the management of chronic and comorbid diseases. Delayed care of diseases has led to poor management and increased progression of diseases. Due to shortages of healthcare resources and the risk of infection and virus transmission, many patients and PCPs have postponed or canceled many outpatient visits and preferred telemedicine.[17] However, medical care delay or avoidance might increase morbidity and mortality risk associated with treatable and preventable health conditions and might contribute to reported excess deaths directly or indirectly related to COVID-19. In addition to this, if routine care avoidance were to be sustained, adults could miss opportunities for management of chronic conditions, receipt of routine vaccinations, or early detection of new conditions, which might worsen outcomes. [18] According to a web-based survey in the USA, administered by Qualtrics, LLC, during June 24-30, 2020, to a nationwide representative sample of U.S. adults aged $\geq 18$ years, an estimated $40.9 \%$ of U.S. adults have avoided medical care during the pandemic because of concerns about COVID-19, including 12.0\% who avoided urgent or emergency care and 31.5\% who avoided routine care. [18] It has specifically impacted the care for Cancer, Cardiovascular diseases, Diabetes, Pregnancy, dialysis, HIV, and other chronic diseases.

To manage chronic and comorbid conditions during the pandemic and avoiding complications due to delayed care it is important to resume primary care services as soon as it is safe and practical, identifying population-based registries of patients with gaps in care, specifically targeting the gaps resulting from COVID-19-related deferrals, and creating options and ensuring insurance coverage for nontraditional lab collection options, such as neighborhood pharmacy, other specimen collection centers, or home testing kits. In addition to this Practices need to emphasize the importance of ongoing prevention screening, monitoring, and treatment to patients who may continue to be fearful about returning to healthcare facilities. [17]

\section{CONCLUSION}

Pandemics are once in a lifetime occurrence and have a profound impact on all areas of life. The COVID-19 Pandemic has further accelerated the impending pandemic of antimicrobial resistance, the epidemic of mental health disorders, and the opioid crisis epidemic. In addition to the above-mentioned impacts of the COVID-19 Pandemic in healthcare, it has also impacted the Economy and Environment. 
As the second wave of COVID-19 Pandemic begins and many countries have resorted to lockdown again to contain the spread of the virus, it is important to remember the lessons we learned during the first wave as we move forward and ensure the continuation of primary health services through telemedicine, management of AMR through stewardship programs, the continuation of de-addiction services and focus on mental health through virtual peer groups, social support, and counseling. The silver lining to this Pandemic is that it has shown the flaws and needs of the healthcare system and society to be worked on so that we as a society can emerge from this Pandemic stronger and kinder.

\section{SOURCES OF FUNDING}

This research received no specific grant from any funding agency in the public, commercial, or not-for-profit sectors.

\section{CONFLICT OF INTEREST}

The author has declared that no competing interests exist.

\section{ACKNOWLEDGMENT}

None.

\section{REFERENCES}

[1] COVID-19: The Hidden Impact on Mental Health and Drug Addiction- Stefania Chiappini1*, Amira Guirguis1,2*, Ann John2, John Martin Corkery1, and Fabrizio Schifano1- Front. Psychiatry, 29 July 2020.

[2] Mental health survey of medical staff in a tertiary infectious disease hospital for COVID-19-Huang JZ1, Han MF1, Luo TD1, Ren AK1, Zhou XP1 - Chinese Journal of Industrial Hygiene and Occupational Diseases, 04 Mar 2020, 38(3):192-195

[3] Anxiety and depression in COVID-19 survivors: Role of inflammatory and clinical predictors - Mario Gennaro Mazzaa, Rebecca De Lorenzo, Caterina Conte, Sara Poletti, Benedetta Vai , Irene Bollettini , Elisa Maria Teresa Melloni , Roberto Furlan, Fabio Ciceri, Patrizia Rovere-Querini , and the COVID-19 BioB Outpatient Clinic Study group, Francesco Benedettia

[4] Petterson S, Westfall J, Miller BF. Projected Deaths of Despair During the Coronavirus Recession. Well, Being Trust (2020) 8:2020. WellBeingTrust.org.

[5] Mental Health and the Covid-19 Pandemic-Betty Pfefferbaum, M.D., J.D., Carol S. North, M.D., M.P.E.- The New England Journal of Medicine-August 06,2020.

[6] Antimicrobial resistance - World Health Organization

[7] How covid-19 is accelerating the threat of antimicrobial resistance- Jeremy Hsu-BMJ 2020

[8] Bacterial and fungal co-infection in individuals with coronavirus: A rapid review to support COVID-19 antimicrobial prescribing- Rawson TM, Moore LSP, Zhu N, Ranganathan N, Skolimowska K, Gilchrist M, et al. Clin Infect Dis. 2020 May 2; ciaa530.

[9] Clinical management of COVID-19 Interim Guidance - May 2020. Geneva: World Health Organization; 2020.

[10] Tackling antimicrobial resistance in the COVID-19 pandemic - Haileyesus Getahun a, Ingrid Smith a, Kavita Trivedi a, Sarah Paulin a \& Hanan H Balkhy b -Bulletin of the World Health Organization 2020

[11] Microbial strategies to reduce pathogens and drug resistance in clinical settings- Caselli E. Hygiene- Microb Biotechnol. 2017

[12] Biocidal agents used for disinfection can enhance antibiotic resistance in gram-negative species- Kampf G Antibiotics (Basel). 2018 Dec 1

[13] The Novel Coronavirus COVID-19 Outbreak: Global Implications for Antimicrobial Resistance - Aimee K. Murray* -Front. Microbiol., 13 May 2020 
[14] Antimicrobial Stewardship at the Core of COVID-19 Response Efforts: Implications for Sustaining and Building Programs - Hasti Mazdeyasna \& Priya Nori \& Payal Patel \& Michelle Doll \& Emily Godbout \& Kimberly Lee \& Andrew J. Noda \& Gonzalo Bearman \& Michael P. Stevens - Current Infectious Disease Reports (2020)

[15] COVID-19 and addiction - Mahua Jana Dubey, Ritwik Ghosh, Subham Chatterjee, Payel Biswas, Subhankar Chatterjee, Souvik Dubey - Diabetes \& Metabolic Syndrome: Clinical Research \& Reviews

[16] Collision of the COVID-19 and Addiction Epidemics- Nora D.Volkow- Annals of Internal Medicine

[17] The Invisible Epidemic: Neglected Chronic Disease Management During COVID-19- Adam Wright PhD1, Alejandra Salazar PharmD2, Maria Miric PhD2, Lynn A. Volk MHS3, Gordon D. Schiff MD2- J Gen Intern Med 35(9):2816-7

[18] Delay or Avoidance of Medical Care Because of COVID-19-Related Concerns — United States, June 2020- Mark É. Czeisler et.al - Weekly / September 11, 2020, / 69(36);1250-1257- CDC. 